\title{
A Use-Case Study on Multi-View Hypothesis Fusion for 3D Object Classification
}

\author{
Panagiotis Papadakis \\ IMT Atlantique Bretagne-Pays de la Loire, LabSTICC UMR 6285, team IHSEV \\ Technopôle Brest-Iroise, France \\ panagiotis.papadakisdimt-atlantique.fr
}

\begin{abstract}
Object classification is a core element of various robot services ranging from environment mapping and object manipulation to human activity understanding. Due to limits in the robot configuration space or occlusions, a deeper understanding is needed on the potential of partial, multiview based recognition. Towards this goal, we benchmark a number of schemes for hypothesis fusion under different environment assumptions and observation capacities, using a large-scale ground truth dataset and a baseline view-based recognition methodology. The obtained results highlight important aspects that should be taken into account when designing multi-view based recognition pipelines and converge to a hybrid scheme of enhanced performance as well as utility.
\end{abstract}

\section{Introduction}

View-based methods for object classification have been shown in latest extensive experiments [4], [16] to outmatch 3D-based shape description methodologies of complete shapes. Such results are particularly encouraging for robotic applications since observing objects from multiple viewpoints is often infeasible either due to robot kinematic constraints or due to the presence of obstacles. On the other hand, it is generally true that not all viewpoints are equally informative in discriminating the class of an object. This is because the discriminative features of an observation taken from a particular viewpoint depend on the intra-class as well as inter-class variability as well the nature of the underlying features that are extracted (cf. Fig 3., [19]). As a consequence, there is an unavoidable bias/utility towards certain viewpoints when classifying objects depending on the shape of the object and its semantic class (cf. Fig. 2, [8] and Fig. 3 , [11]). Therefore, while an integral inspection of an object may not be necessary for correct classification, we can expect that in the worst case two or more observations will be required in order to capture the most discriminative object parts.

Most earlier works addressing multi-view object classification are application oriented with predetermined assumptions. The authors of [8] exploit the viewpoint bias of detectors only empirically by adopting the shape description method with the highest influence to viewpoint variability and mostly evident among positive and negative examples. To fuse the classification outputs obtained from the total set of observations they apply the naive Bayes criterion, also applied in their consecutive work [9].

In the work of Becerra et al. [2] a POMDP formulation for confirming the class of an object is proposed which integrates information on robot location and the output of an object detector. A solution is found via stochastic dynamic programming for a fixed time horizon, but only after assuming a particular class for the object of interest before trying to optimize the detection score while performing training and testing on the same object. This makes that approach unsuited when the objective is to confirm one among many possible classes and on different test and train objects.

Potthast et al. [12] also adopt a POMDP formulation for joint category and viewpoint classification of an object by further incorporating feature selection on interleaved steps of local and global optimization. Since their main goal is to extract the optimal category and viewpoint at the last observation of an object with no concern on the consistency along the entire observation sequence, state transitions among categories are not excluded which could lead to unreasonable results.

In [1] the authors propose a model for active object classification and pose estimation that jointly considers sensor movement and decisional cost. They are also based on a POMDP formulation, yet its practical utility is limited because successive observations are considered independent and state transitions among object categories are also allowed. Their experiments also appear to be limited by considering only 1 object category at a time.

Finally, in the work of Patten et al. [11], class and pose 
are estimated separately and by assuming observation independence for both problems. The class distribution is obtained by Bayesian updates while pose by the best alignment among all observations and the best match given by the classifier, through Iterative Closest Point (ICP) registration.

Overall, due to differences in the applications, assumptions and detectors among earlier works it is not straightforward to derive conclusions on the conditions under which multi-view classification is beneficial compared to singleview object classification.

In this work, we examine the performance of multi-view hypothesis fusion for $3 \mathrm{D}$ object classification both under observation dependence or independence and in consideration of the robot observational capacities. Under observation dependency, we detail how multiple classes can be hypothesized by firstly evaluating the viewpoint sequence estimation problem for every hypothesized class and secondly by assigning the class with the highest overall probability. After comparison against alternative fusion schemes under observation independence, the experiments highlight that it proves consistently beneficial with the increase of distinct observations, in contrast to observation dependence which proves superior only early in the observation sequence.

The remaining of the article is organized as follows. In Section 2 we formulate the problem under consideration, in Section 3 we describe different hypothesis fusion schemes along with the conditions and assumptions that are applicable for each case and in Section 4 we evaluate crucial aspects of multi-view based object classification on a public dataset.

\section{Problem Description}

We treat the problem of optimal classification of static or dynamic 3D objects from sequences of observations acquired from multiple viewpoints by a mobile sensor. A ground truth of labelled object templates observed from various viewpoints is assumed available where each annotated training sample is a tuple composed of the semantic class of the object, the observed viewpoint and the corresponding feature vector. Considering the observation viewpoint as part of the ground truth implies that objects that belong to the same semantic class of the ground truth must share a fixed canonical pose, namely, they are aligned to each other as in the case of ModelNet [20] or CAPOD datasets [10].

When exploring a new environment, i.e. during testing, both the semantic class as well as the pose of an object are unknown and thus the challenge resides in devising them from the collected observations. In the context of a robotic application, we are generally more interested in hypothesizing the semantic class and secondarily the pose since the latter can be ambiguous or even absent due to shape symmetry across object views. Object classification will therefore be used as the final performance measure criterion.

To address the outlined problem, we may rely on: (i) a capacity to hypothesize the semantic class and the observed viewpoint of an object based on a single frame/observation and (ii) an approximate sensor localization capacity. The first capacity allows only for joint consideration of different observations under the assumption that they are independent while the second capacity allows to enforce dependency between successive observations. Upon presenting these capacities in sections 2.1,2.2 we then detail the fusion schemes that can be considered depending on assumptions in Section 3 .

\subsection{Single Observation Model}

A feature vector classification method is employed (see Sec. 4) which classifies a given feature vector $\mathbf{x}$ describing an object $o_{\mathbf{x}}$ to one of $|I|$ classes $\omega_{i} \in I, i \in\{1,2, \ldots|I|\}$ based on the corresponding probability $P\left(\omega_{i} \mid \mathbf{x}\right)$. A feature vector corresponds to the global shape descriptor extracted by observing an unknown object from an unknown viewpoint. If we assert that an object $o_{\mathbf{x}}$ belongs to a specific class $\omega_{i}$, we write $o_{\mathbf{x}}=\omega_{i}$.

For the evaluation of the posterior probability $P\left(\omega_{i} \mid \mathbf{x}\right)$, a nearest neighbor classification scheme is adopted which sets the probability as inversely proportional to the matching distance between $\mathbf{x}$ and the nearest neighbor that belongs to $\omega_{i}$, namely:

$$
\begin{gathered}
P\left(\omega_{i} \mid \mathbf{x}\right) \propto \frac{1}{\operatorname{dist~}_{N N}\left(\mathbf{x}, \omega_{i}\right)} \\
\operatorname{dist}_{N N}\left(\mathbf{x}, \omega_{i}\right)=\min _{\mathbf{y} \in \mathbf{Y} \mid o_{\mathbf{y}}=\omega_{i}} \operatorname{dist}(\mathbf{x}, \mathbf{y})
\end{gathered}
$$

where $\mathbf{Y}$ is the total set of feature vectors of the ground truth database that are compared against $\mathbf{x}$ and $\operatorname{dist}(.,$.$) is$ the distance function for a pair of feature vectors.

The total number of possible classes is therefore $|I|=$ $|V| \cdot|S|$ where $|V|$ is the cardinality of the set of viewpoints $V$ from which each object is observed and $|S|$ the cardinality of the set of object semantic classes $S$. It follows that each $\omega_{i}$ is associated with a particular object viewpoint $\theta_{v}=(v-1) 2 \pi /|V|, v \in\{1,2, \ldots|V|\}$ of a particular semantic class $c_{s}, s \in\{1,2, \ldots|S|\}$, which can be equivalently denoted as $\omega_{v s}$ through the linear mapping $i=v+(s-1)|V|$. We will hereafter employ the two notations $\omega_{i}$ and $\omega_{v s}$ interchangeably for convenience depending on the context. Fig. 1 depicts the underlying notations with a representative object of semantic class armchair.

\subsection{Sensor Localization and Transition Model}

We consider a mobile robotic sensor with the capability of aproximate 2D localization around an object of interest, e.g. following the approach described in [6]. The location of the sensor is used for measuring its displacement across 


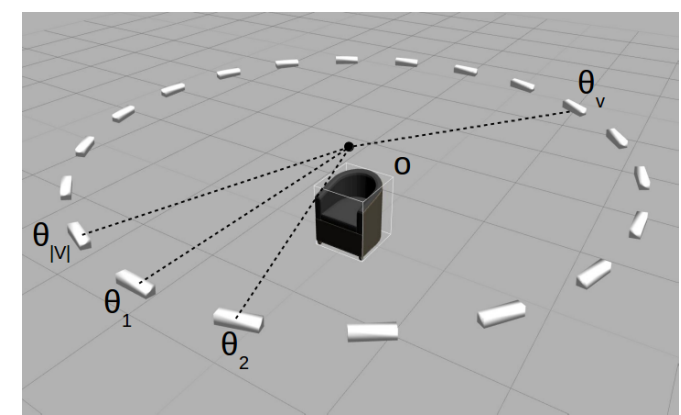

Figure 1. Example object and observation configuration

succesive observations and in turn, obtain the expected transition/shift in the observed object pose. Considering a series of observations where $k \in\{2,3 \ldots, N\}$ denotes the observation step, we can readily obtain the transition probability $p\left(\omega_{v(k) s(k)} \mid \omega_{v(k-1) s(k-1)}\right)$, where $\omega_{v(k) s(k)}$ is one of the possible classes that can be appointed to an object at the $k^{t h}$ observation step, i.e. $v(k) \in\{1,2, \ldots|V|\}$ and $s(k) \in\{1,2, \ldots|S|\}$.

Recalling subsection 2.1, each class $\omega_{i}$ can be equivalently referred as $\omega_{v s}$ where $v$ and $s$ correspond to a viewpoint and a semantic object class respectively. Evidently, when observing a particular object there can only be transitions between viewpoints and not among semantic classes. Therefore, we simplify the transition probability as:

$$
p\left(\omega_{v(k) s} \mid \omega_{v(k-1) s}\right)=\mathcal{N}\left(\Delta \alpha ; \mu_{\Delta \theta_{k}}, \sigma\right)
$$

where $\mu_{\Delta \theta_{k}}=\theta_{v(k)}-\theta_{v(k-1)}$ is the angle between viewpoints $v(k)$ and $v(k-1), \sigma$ corresponds to the standard deviation that quantifies the uncertainty of the sensor localization and $\Delta \alpha$ the angular displacement of the sensor with respect to the object between observations $k-1$ and $k$.

\section{Fusion schemes}

\subsection{Hypothesis Fusion based on Observation De- pendency}

We first investigate the possibility of fusing the total number of obtained hypotheses by assuming dependency among observations, which implies using both capacities described in Sections 2.1, 2.2 Given a sequence of object observations $\mathbf{x}_{k}$, the optimal class is obtained as:

$$
\omega_{i(N)}^{*}=\underset{\omega_{i(N)}}{\operatorname{argmax}} D_{\max }\left(\omega_{i(N)}\right)
$$

where $D_{\max }\left(\omega_{i(N)}\right)$ is the maximum decision score that can be deduced if class $\omega_{i(N)} \in I$ is matched to the underlying object.

The above problem can be formulated as a hidden markov model (HMM) where $\omega_{i(k)}$ are the possible, hidden states along the observation sequence and $\mathbf{x}_{k}$ are the corresponding observations. In this context, the aggregated decision cost at time step $k$ is given by:

$$
D\left(\omega_{i(k)}\right)=\sum_{r=1}^{k} d\left(\omega_{i(r)}, \omega_{i(r-1)}\right)
$$

where $d\left(\omega_{i(r)}, \omega_{i(r-1)}\right)$ is the cost that is associated to a transition between succesive states $\omega_{i(r)}$ and $\omega_{i(r-1)}$. Although the term cost may seem counterintuitive since we seek to maximize eq. (4), it is adopted here for the sake of correspondence to the literature (cf. [17], Ch. 9) which further holds true for the chosen notations.

Recalling that when observing a particular object the only possible state transitions concern transitions between viewpoints and not among semantic classes, it follows that eq. (5) can be expressed as:

$$
D\left(\omega_{i(k)}\right)=D\left(\omega_{v(k) s}\right)=\sum_{r=1}^{k} d\left(\omega_{v(r) s}, \omega_{v(r-1) s}\right)
$$

where the decisional transition cost $d(.,$.$) is defined as:$

$d\left(\omega_{v(k) s}, \omega_{v(k-1) s}\right)=\ln \left(p\left(\omega_{v(k) s} \mid \omega_{v(k-1) s}\right) \cdot p\left(\mathbf{x}_{k} \mid \omega_{v(k) s}\right)\right)$

where $p\left(\omega_{v(k) s} \mid \omega_{v(k-1) s}\right)$ is obtained by eq. 3) and the likelihood function $p\left(\mathbf{x} \mid \omega_{i}\right)$ introduced in eq. (7) is obtained by applying Bayes rule and assuming equal $a$ priori class probabilities. In other words, we do not assume that certain object classes are more likely to appear than others, although such treatment could be employed when the surrounding spatial context can be accounted for (cf. [5] and [7]). In this way, we obtain that $p\left(\mathbf{x} \mid \omega_{i}\right) \propto P\left(\omega_{i} \mid \mathbf{x}\right) / p(\mathbf{x})$ which makes the calculation of $p(\mathbf{x})$ redundant in the evaluation of the cost in eq. (7). Eventually, the maximization of the aggregated cost $D$ is efficiently calculated via dynamic programming by virtue of Bellman's principle and by employing the Viterbi algorithm [18].

\subsection{Hypothesis Fusion based on Observation Inde- pendence}

Observation independence is assumed in the case of no sensor localization capacity or whenever we cannot assume that objects remain strictly static along the entire set of observations, therefore all state transitions are equiprobable. Under such conditions, we examine a number of possible hypothesis fusion schemes presented as follows.

\subsubsection{Equiprobable Transitions}

The first scheme can be considered as a subcase of the previous by setting the transition probability to be uniformely distributed, i.e. $p\left(\omega_{v(k) s} \mid \omega_{v(k-1) s}\right)=1 /|V|$. This implies that the transitional decision cost of eq. (7) depends only on the observation likelihood while the optimal class $\omega_{i(N)}^{*}$ is obtained in the same manner using Viterbi algorithm. 


\subsubsection{Maximum Similarity}

In this scheme, we hypothesize as class of the object the one corresponding to the viewpoint observation with the maximum probability, namely:

$$
\left.\omega_{i(N)}^{*}=\underset{\omega_{i(k)}}{\operatorname{argmax}} P\left(\omega_{i(k)} \mid \mathbf{x}\right)\right)
$$

where $P\left(\omega_{i(k)}\right)$ is calculated using eq. 11 .

\subsubsection{Maximum Certainty}

We hypothesize as class of the object the classification result of the viewpoint observation with the maximum classification certainty, namely:

$$
\begin{gathered}
\omega_{i(N)}^{*}=\underset{\omega_{i(k)}^{*}}{\operatorname{argmin}} H_{i(k)} \\
\omega_{i(k)}^{*}=\underset{i}{\operatorname{argmax}} P\left(\omega_{i(k)} \mid \mathbf{x}\right) \\
H_{i(k)}=-\sum_{i} P\left(\omega_{i(k)} \mid \mathbf{x}\right) \log P\left(\omega_{i(k)} \mid \mathbf{x}\right)
\end{gathered}
$$

and certainty is quantified via Shannon's information entropy.

\section{Results}

We performed a comparative evaluation of the aforementioned approaches within the Princeton ModelNet10 [20] dataset which contains 4899 objects distributed into 10 common indoor classes, in order, bathtub, bed, chair, desk, dresser, monitor, night stand, sofa, table and toilet. These categories correspond to the top 10 most common indoor object categories according to [21]. Example 3D objects for each category are shown in Fig. 2

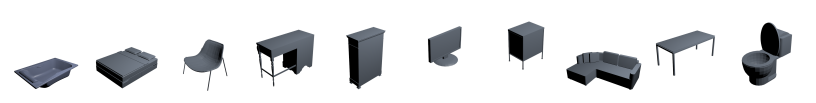

Figure 2. Example 3D objects from ModelNet10 dataset categories

This dataset is particularly suited in the context of a mobile robotic sensor since the pose of each synthetic object coincides with its expected upright orientation in the real world, which in turn allows the adoption of a fixed sensing configuration. For our experiments, we set $M=20$ camera viewpoints uniformly distributed around each object at a sensor pose that is orientated versus the centroid of the coordinate system at a fixed pitch angle. This testing configuration is equivalent to related works on multi-view object classification such as [16], [12] or [4] wherein a roving sensor observes a query object from different viewpoints and estimates its class and possibly its pose. Nevertheless,

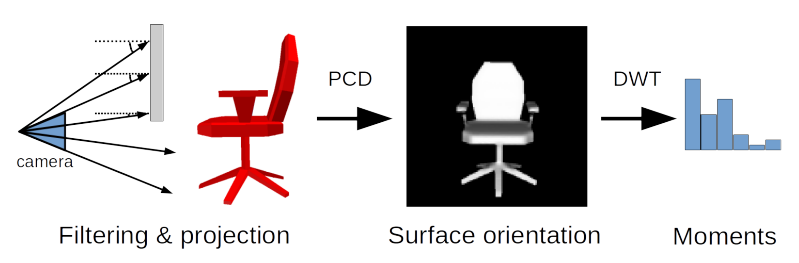

Figure 3. Baseline method for single observation model

Table 1. Comparative classification performance of baseline single view observation model against state-of-the-art methods

\begin{tabular}{|l|l|l|}
\hline & Precision \% & Recall \% \\
\hline Baseline & 71.4 & 70.9 \\
\hline ESF [19] & 73.6 & 72.8 \\
\hline VFH [13] & 69.9 & 69.1 \\
\hline ROPS [3] & 58.3 & 57.7 \\
\hline
\end{tabular}

the scope of our evaluation is different in that we do not seek to compare the performance among different singleview based object recognition methods but among decision fusion schemes based on a common, baseline single-view method.

The baseline approach that we adopt here for the single observation model assumed in Sec. 2.1 is composed of the following steps. First, a bilateral filter is applied on a depth buffer image acquired by simulating a perspective projection of the object using as intrinsic camera parameters those corresponding to a Kinect V1 sensor. The smoothed organized point cloud (PCD) is then used to compute the surface orientation at each sensed point, producing a surface orientation image. That image is finally encoded by a multi-scale 2D discrete wavelet transform (DWT) whose coefficients are used to extract a set of central moments (mean, standard deviation and skewness) for each distinct wavelet sub-band and scale. To compare two feature vectors, the Canberra distance score is employed. This feature extraction procedure is summarized in Fig. 3 As a reference for the general performance of our baseline single observation model, we further provide in Table 1 its comparative performance to three other state-of-the-art singleview based object descriptors in terms of precision and recall. The compared methods are: (i) Ensemble of Shape Functions (ESF) [19], (ii) Rotational Projection Statistics (RoPS) [3] and (iii) VFH (Viewpoint Feature Histograms) [13], whose implementations are available with PCL (PointCloud Library) [14]. These results suggest that the adopted baseline observation model is representative of the state-ofthe-art performance.

To evaluate performance among hypothesis fusion schemes, we calculate the precision and recall scores for each object class and perform leave-one-out experiments by simulating sensor observations from randomly chosen 
viewpoints and $k \in\{2,3, \ldots, 6\}$. We perform a total of 4 independent runs for each $k$ and fusion scheme and finally obtain the average.

We abbreviate the compared hypothesis fusion schemes as follows: HMM-dep (Sec. 3.1), HMM-indep (Sec. 3.2.1), MAXS (Sec. 3.2.2) and MAXC (Sec. 3.2.3). In Fig. 4 we demonstrate the respective results for each class of the dataset, for both precision and recall scores. For the trivial case of 1 observation being considered, since there is no hypothesis fusion all schemes attain the same performance. When multiple observations are performed for $(k=2,3, \ldots)$ the presented results are instructive in a number of points.

First of all, we observe that the consideration of supplementary viewpoint observations contributes almost always in the increase of classification performance (with the exception of the desk Recall and the dresser precision when using the MAXC criterion). The first 3 observations appear to be in general those which contribute the most while performance seems to stabilize afterwards with very little additional benefit. Furthermore, it is clear that the average macroscopic performance for schemes MAXS and MAXC is clearly inferior compared to the other alternatives. This strongly suggests that single-view based object classification cannot alone surpass the performance of multi-view classification. If this is the case then this result may appear contradictory to the results presented in [15] where view-based methods surpass entire 3D object discrimination methods.

An explanation to this contradiction emerges via the comparison between the schemes HMM-dep and HMMindep. Considering observation dependence via HMM-dep is consistently beneficial when $k=2$ observations, while for more observations the performance seems to stabilize in an abrupt way and sometimes even decrease (see dresser Recall, sofa Recall, bed Precision, chair Precision, toilet Precision). Conversely, considering observation independence via HMM-indep, performance is always favoured by the consideration of supplementary observations and convergence is smoother and in turn, more stable. Furthermore, HMM-indep surpasses HMM-dep in the majority of classes both in terms of precision and recall while achieving very close performance to HMM-dep in the remaining cases.

The above observations leads us to the principal outcome of the experiments, namely, that multi-view object classification is strongly influenced on the underlying assumptions (i.e. viewpoint dependency) and occasionally by the object category. The fact that viewpoint independence appears to generally be more beneficial than considering dependency, means that there may be classes easier to be confused if they exhibit similar visual characteristics when observed from the same viewpoints. In such cases, observation independence allows an observation to be matched to an arbitrary
Table 2. Average macroscopic performance of hypothesis fusion schemes as a function of supplementary observations

\begin{tabular}{|l|c|c|c|c|c|}
\hline & \multicolumn{5}{|c|}{ Recall/Precision per number of observations } \\
\hline $\begin{array}{l}\text { Fusion } \\
\text { schemes }\end{array}$ & 2 & 3 & 4 & 5 & 6 \\
\hline HMM-dep & $\mathbf{7 8 . 8 / 7 9 . 7}$ & $79.9 / 80.8$ & $80.7 / 81.5$ & $81.3 / 82.2$ & $81.8 / 82.7$ \\
\hline HMM-indep & $77.8 / 78.7$ & $\mathbf{8 1 . 4 / 8 2 . 9}$ & $\mathbf{8 2 . 1 / 8 3 . 6}$ & $\mathbf{8 2 . 6 / 8 4 . 2}$ & $\mathbf{8 3 . 1 / 8 4 . 6}$ \\
\hline MAXS & $73.4 / 73.8$ & $75 / 75.6$ & $75.4 / 76.1$ & $76 / 76.7$ & $76 / 76.8$ \\
\hline MAXC & $72.9 / 73.4$ & $73.9 / 74.5$ & $74.1 / 74.6$ & $74.5 / 75.1$ & $74.3 / 74.8$ \\
\hline
\end{tabular}

view of an object, which means that objects can be deemed similar if they generally share similar visual characteristics without explicitly requiring spatial correspondence.

Driven by this analysis, we can deduce a hybrid fusion scheme that combines viewpoint dependence with viewpoint independence, by applying HMM-dep for $k=2$ and HMM-indep for $k>2$. The average macroscopic performance of all schemes presented in Table 2 clearly shows the performance difference between $k=2$ with observation dependence and $k>2$ with observation independence. This means that optimal multi-view object classification should be initially sought by spatially corresponding visual similarity between pairs of objects and secondarily by random, non spatially corresponding visual similarity. The latter element being the determining factor in disambiguating semantically different classes with increased visual correspondence.

This theoretically optimal scheme is also practically appealing in scenarios involving robots that explore an environment and look for objects. It implies that accurate localization of the robotic sensor with respect to the object in order to enforce viewpoint dependence is only important at the early stage of observation. In other words, an object would be assumed to be static for only two consecutive observations while in the sequel the robot would deliberately consider that the object may have moved or that sensor localization is less accurate and therefore enforce observation independence. In the worst-case where the object moved among the first and second observation then as shown by Fig. 4 and Table 2, the performance difference between HMM-dep and HMM-indep would be trivial.

\section{Conclusions}

This work has presented an elaborate study targeting the application of multi-view hypothesis fusion schemes for the purpose of 3D object classification. We evaluated different fusion schemes that are distinguished by the underlying assumptions regarding the objects (static or dynamic) and the sensor observations (dependency), on a common benchmark. The performed experiments highlight the ex- 

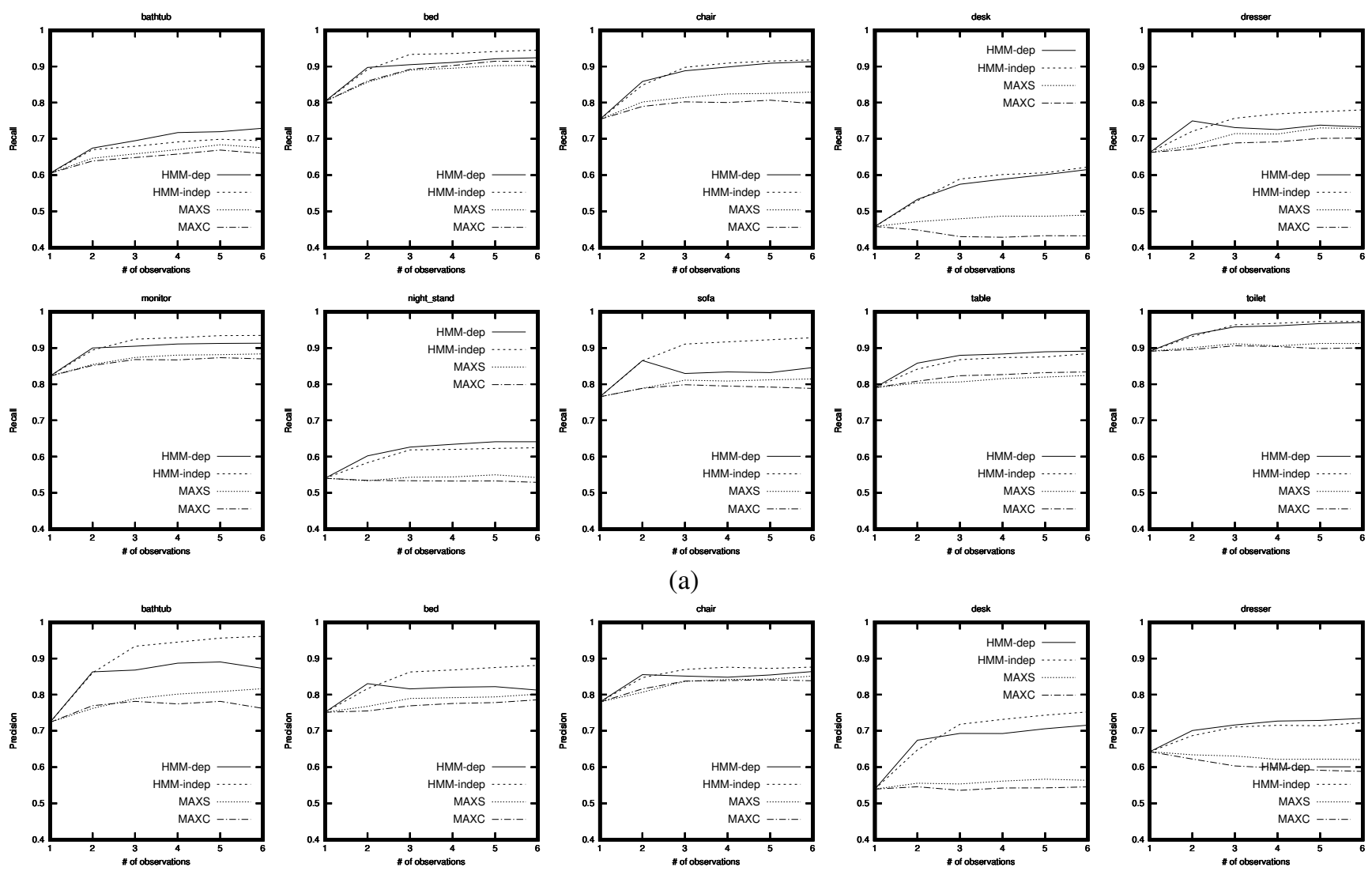

(a)
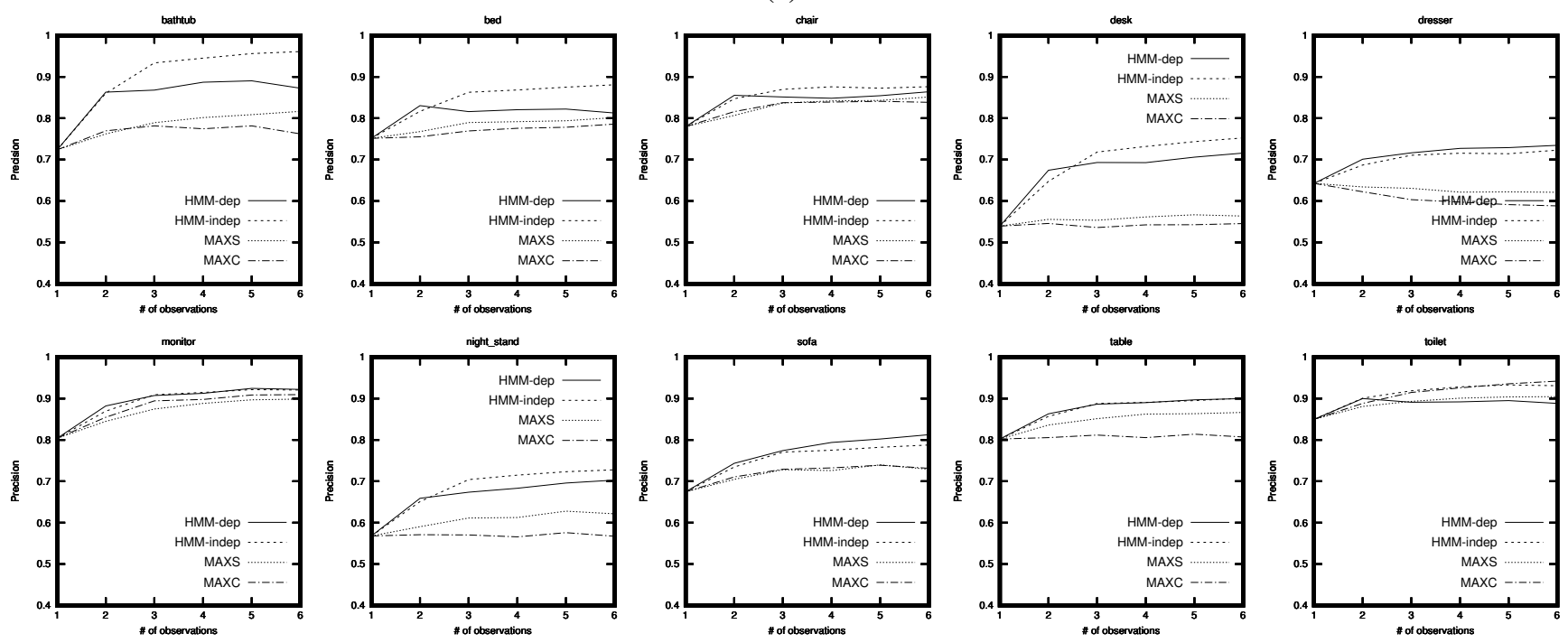

(b)

Figure 4. Comparison of multi-view hypothesis fusion schemes; (a) Recall and (b) Precision

istence of a certain trade-off in the way that multiple observations should be considered for optimal classification performance.

In future work, it would be interesting to extend these experiments by alternating other parameters such as alternative baseline single observations models, varying sensing configurations or bigger object collections, which would allow more solid conclusions. Finally, as the analysis was solely based on 3D shape with no consideration on texture characteristics, subsequent work could emphasize on photometric and color data of real objects.

\section{Acknowledgements}

The author would like to acknowledge the contributions of David Filliat and Céline Craye for constructive discussions on the exploitation of the HMM model and the partial support of the project COMRADES (COordinated MultiRobot Assistance Deployment in Smart Spaces) - IMT Fonds Santé.

\section{References}

[1] N. Atanasov, B. Sankaran, J. L. Ny, G. J. Pappas, and K. Daniilidis. Nonmyopic view planning for active object classification and pose estimation. IEEE Transactions on Robotics, 30(5):1078-1090, 2014. 
[2] I. Becerra, L. M. Valentn-Coronado, R. Murrieta-Cid, and J.-C. Latombe. Reliable confirmation of an object identity by a mobile robot: A mixed appearance/localization-driven motion approach. The International Journal of Robotics Research, 2016.

[3] Y. Guo, F. Sohel, M. Bennamoun, M. Lu, and J. Wan. Rotational projection statistics for $3 \mathrm{~d}$ local surface description and object recognition. Int. Journal of Computer Vision, 105(1):63-86, 2013.

[4] B.-S. Hua, Q.-T. Truong, M.-K. Tran, Q.-H. Pham, A. Kanezaki, T. Lee, H. Chiang, W. Hsu, B. Li, Y. Lu, H. Johan, S. Tashiro, M. Aono, M.-T. Tran, V.-K. Pham, H.-D. Nguyen, V.-T. Nguyen, Q.-T. Tran, T. V. Phan, B. Truong, M. N. Do, A.-D. Duong, L.-F. Yu, D. T. Nguyen, and S.-K. Yeung. RGB-D to CAD Retrieval with ObjectNN Dataset. In Eurographics Workshop on 3D Object Retrieval, 2017.

[5] F. Husain, H. Schulz, B. Dellen, C. Torras, and S. Behnke. Combining semantic and geometric features for object class segmentation of indoor scenes. IEEE Robotics and Automation Letters, 2(1):49-55, 2017.

[6] S. Kohlbrecher, J. Meyer, O. von Stryk, and U. Klingauf. A flexible and scalable slam system with full $3 \mathrm{~d}$ motion estimation. In IEEE Int. Symp. on Safety, Security and Rescue Robotics, 2011.

[7] L. Kunze, K. K. Doreswamy, and N. Hawes. Using qualitative spatial relations for indirect object search. In IEEE Int. Conf. on Robotics and Automation, 2014.

[8] D. Meger, A. Gupta, and J. J. Little. Viewpoint detection models for sequential embodied object category recognition. In IEEE Int. Conf. on Robotics and Automation, 2010.

[9] D. Meger and J. J. Little. Mobile 3d object detection in clutter. In IEEE Int. Conf. on Intelligent Robots and Systems, 2011.

[10] P. Papadakis. The Canonically Posed 3D Objects Dataset. In Eurographics Workshop on 3D Object Retrieval, 2014.

[11] T. Patten, M. Zillich, R. Fitch, M. Vincze, and S. Sukkarieh. Viewpoint evaluation for online 3-d active object classification. IEEE Robotics and Automation Letters, 1(1):73-81, 2016.

[12] C. Potthast, A. Breitenmoser, F. Sha, and G. S. Sukhatme. Active multi-view object recognition: A unifying view on online feature selection and view planning. Robotics and Autonomous Systems, 84:31 - 47, 2016.

[13] R. Rusu, G. Bradski, R. Thibaux, and J. Hsu. Fast 3d recognition and pose using the viewpoint feature histogram. In Int. Conf. on Intelligent Robots and Systems, 2010.

[14] R. B. Rusu and S. Cousins. 3D is here: Point Cloud Library (PCL). In Int. Conf. on Robotics and Automation, 2011.

[15] K. Sfikas, T. Theoharis, and I. Pratikakis. Exploiting the PANORAMA Representation for Convolutional Neural Network Classification and Retrieval. In Eurographics Workshop on 3D Object Retrieval, 2017.

[16] H. Su, S. Maji, E. Kalogerakis, and E. G. Learned-Miller. Multi-view convolutional neural networks for $3 \mathrm{~d}$ shape recognition. In Int. Conf. on Computer Vision, 2015.

[17] S. Theodoridis and K. Koutroumbas, editors. Pattern Recognition. Academic Press, 2003.
[18] A. Viterbi. Error bounds for convolutional codes and an asymptotically optimum decoding algorithm. IEEE Transactions on Information Theory, 13(2):260-269, 1967.

[19] W. Wohlkinger and M. Vincze. Ensemble of shape functions for $3 \mathrm{~d}$ object classification. In Int. Conf. on Robotics and Biomimetics, 2011.

[20] Z. Wu, S. Song, A. Khosla, F. Yu, L. Zhang, X. Tang, and J. Xiao. 3d shapenets: A deep representation for volumetric shapes. In Conf. on Computer Vision and Pattern Recognition, 2015.

[21] J. Xiao, J. Hays, K. A. Ehinger, A. Oliva, and A. Torralba. Sun database: Large-scale scene recognition from abbey to zoo. In IEEE Int. Conf. on Computer Vision and Pattern Recognition, pages 3485-3492, 2010. 